\title{
Fashion Supply Chain Optimization: A Linear Programming Model
}

\author{
Sara Shoarinejad ${ }^{\mathrm{a}}$, Amin Shoari Nejad $^{\mathrm{b}}$, \\ ${ }^{a}$ Department of Industrial Engineering, K. N. Toosi University of Technology, Tehran, Iran \\ ${ }^{\mathrm{b}}$ Hamilton Institute, Deparment of Mathematics and Statistcs, Maynooth University, Kildare, Ireland
}

\begin{abstract}
Fashion supply chain management is challenging due to demand uncertainty, short product life cycle, fierce global competition, increasing expectations for diversity in production, and keeping pace with the current and emerging trends. In this paper, a brief review of the published works in the field of fashion and clothing supply chain is presented and an integrated linear model is developed to minimize the costs of a fashion brand, including production and logistics costs, enabling the firm to make better decisions with regard to its production and replenishment policies and to respond in a reasonable time to customers demands for various designed items. We show that our model can successfully account for the complex structure of the supply chain consisting of designers, manufacturers, distributors, and stores. The model is validated using the General Algebraic Modeling System (GAMS) with the data collected from an Iranian fashion brand.
\end{abstract}

Key words: Fashion Supply Chain Management; Operations research; Optimization; Mathematical Modelling.

\section{Introduction}

The fashion industry as a whole can consist of design, procurement of raw materials, production, distribution, marketing and sales. Mass production of clothes started in mid-19th century when producers started producing cloths that didn't need the tailor to take measures for every customer individually. However, fashion did not become an industry until the 20th century when the network of local tailors transformed into bigger production businesses. After world war II textile factories and clothing businesses experienced higher development due to higher demands and cultural and societal changes which eventually resulted in less regularization and more diversity in the clothing and fashion industry. At the beginning of the 20th century, significant changes in the fashion business such as higher attention to creativity in design, European fashion houses, technological development such as sewing machines, the emergence of capitalism, systematic development of factories and retailers made the fashion industry bigger and more powerful $[9,2]$.

Email addresses: sarashoarinejad@gmail.com (Sara Shoarinejad), amin.shoarinejad@gmail.com (Amin Shoari Nejad).
Today fashion industry is bigger than ever and in order to remain in the business it is very important to efficiently manage the fashion supply chain especially in the presence of different players such as suppliers, distributors and producers that need to collaborate in harmony in order to meet the market demand. Fashion supply chain management requires considering the industry as a complex interconnected system in which businesses need to try balancing supply and demand, minimizing costs, maximizing profit, managing risk, and unifying their processes and their associated information flows for optimizing their supply chain and obtaining competitive advantage $[1,11]$.

In this paper, we aim at developing a mathematical model that takes into account demand for multiple fashion items in different stores and helps businesses to decide about their production quantities and distribution plans while minimizing their costs.

The paper is organized as follows. In section 2, a summary of previous related works is provided. In section 3 , we describe the problem that we try to solve. In section 4, we explain our modelling approach. In section 5 we discuss the model validation and results. In section 6 , we discuss the implications of our method and how our model can help decision makers. We summerize the paper and conclude in 7 . 


\section{Literature review}

[3] studied a flexible pricing scheme that allows the price to vary depending on customer. Their goal was to find an optimal solution using a unified model that considers the flexibility between the start and the end time of production and acceptance/rejection of the orders. They used a mixed-integer programming and a heuristic method to solve the problem and showed the significant effect of unified production on profit considering other decisions of the supply chain.

[7] focused on fashion products under deterministic demand, economic order quantity (EOQ) and limited replenishment. Fashion retailers usually use dynamic pricing (e.g. offering discount) to deplete their stocks. They found the optimal order quantity and order point in order to maximize profit and showed the effect of them on demand by considering the customer saving price (the minimum price that a customer is willing to pay). They utilized discrete and dynamic pricing schemes and alternated between them multiple times in order to satisfy customers demand in different periods and minimized the inventory cost.

[10] introduced a system dynamics model to analyze the fashion supply chain. They evaluated the effect of work duration and transportation delays on the performance of a fast fashion supply chain including three levels of producer, distributor and retailer. They showed how each level is affected by the delays.

[8] developed a causal loop diagram for fashion supply chain. They divided the supply process into two parts: pre sale season and the sale season. The former includes collection planning, demand forecasting, purchase, logistics and replenishment and the latter includes balancing supply and demand.

[5] focused on the problem of determining initial order quantity and replenishment of a retail product to minimize the opportunity cost, cost of returned products and expired inventory. They framed the problem into a two-step stochastic dynamical programming model and suggested a novel heuristic method to find the optimal solution. They showed that their solution could double the profit.

[6] in collaboration with ZARA, designed and tested a support system model for replenishment and distribution. Their model is data-driven and is designed to update demand forecasting and allocate inventory through time using dynamical optimization methods.

Fast fashion is a competitive area in the fashion industry and new and diverse trends, highly uncertain demand, and high inventory are some of its important characteristics. Therefore, in this area, decision makers should have
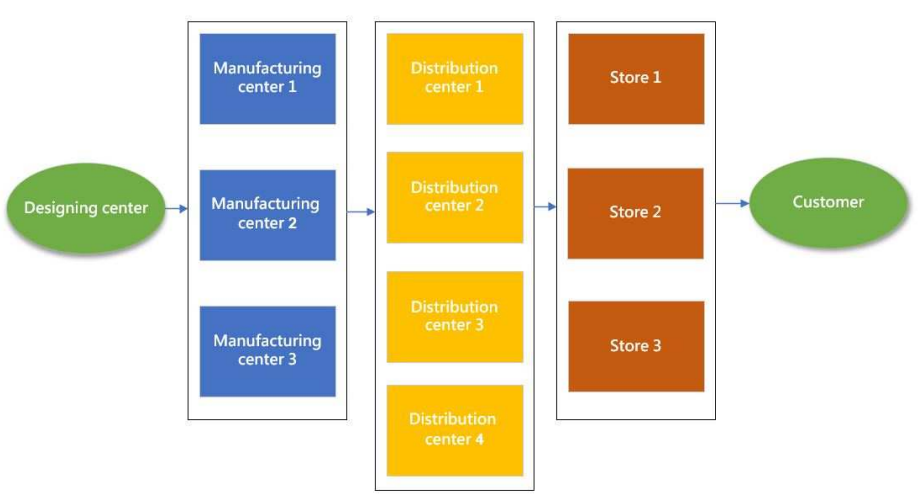

Fig. 1. Schematic of the fashion supply chain.

high operations flexibility to respond to demand in a timely manner. [4] developed an optimization model for a supply chain of a fashion retailer for a fast response to demand and having an optimized replenishment policy. Their model is cable of calculating the optimal quantity that needs to be transferred between stores in order to meet the demand in different locations. They suggested that their model can help international retailers to make better decisions for replenishing their stocks according to feedback from the market and sales data.

\section{Problem definition}

In this section we outline the problem. The goal is to develop a model for a multilevel fashion supply chain enabling fast response to customers demands. We suggest a model considering a direct supply chain and we aim at developing a decision support system for production and replenishment quantities. We use data provided by Queen Manto, a fashion brand based in Iran. Our suggested model could be an extension to the [4] by considering four levels of fashion supply chain instead of one. The supply chain of our problem includes designing center, manufacturing centers, distribution centres and stores. The functionality of each level are as follows:

(1) Designing center: Determining the kind and quantities of different designs to be produced by the manufacturing centers.

(2) Manufacturing centers: Producing the placed orders and sending them to the distribution centers.

(3) Distribution centers: Products are stored in these centers before being sent to stores.

(4) Stores: Products received from the distribution centers are sold to customers in stores.

\section{Modelling procedure}

In this section we explain the details of our modelling approach for the supply chain schematically shown in figure 1 . We consider the total cost, including fixed and 
Table 1. Nomenclature used in the model.

\begin{tabular}{|c|c|}
\hline Indices & Description \\
\hline$i: i \in\{1,2, \ldots, I\}$ & designs \\
\hline$j: j \in\{1,2, \ldots, J\}$ & manufacturers \\
\hline$k: k \in\{1,2, \ldots, K\}$ & distribution centers \\
\hline$e: e \in\{1,2, \ldots, E\}$ & stores \\
\hline Decision variables & Description \\
\hline $\begin{array}{l}\operatorname{trans}_{i j k} \\
\text { trans }_{i k e} \\
\quad x_{i j}\end{array}$ & $\begin{array}{c}\text { number of item i to be sent from manufacturer } \mathrm{j} \text { to distribution center } \mathrm{k} \\
\text { number of item } \mathrm{i} \text { to be sent from distribution center } \mathrm{k} \text { to store e } \\
\text { number of item } \mathrm{i} \text { to be produced by manufacturer } \mathrm{j}\end{array}$ \\
\hline Model parameters & Description \\
\hline$c_{i j}$ & cost of producing item i by manufacturer $\mathrm{j}$ \\
\hline$c t f_{j k}$ & fixed cost of sending item $\mathrm{i}$ from manufacturer $\mathrm{j}$ to distribution center $\mathrm{k}$ \\
\hline$c t v_{j k}$ & variable cost of sending item $\mathrm{i}$ from manufacturer $\mathrm{j}$ to distribution center $\mathrm{k}$ \\
\hline$c t f_{k e}$ & fixed cost of sending item i from distribution center $\mathrm{k}$ to store $\mathrm{e}$ \\
\hline$c t v_{k e}$ & variable cost of sending item i from distribution center $\mathrm{k}$ to store e \\
\hline $\operatorname{cap}_{i}$ & capacity of manufacturer $\mathrm{j}$ \\
\hline $\operatorname{cap}_{k}$ & capacity of manufacturer $\mathrm{k}$ \\
\hline $\operatorname{cap}_{e}$ & capacity of store e \\
\hline $\operatorname{dem}_{i e}$ & demand for item $\mathrm{i}$ in store e \\
\hline dist $_{j k}$ & distance from manufacturer $\mathrm{j}$ to distribution center $\mathrm{k}$ \\
\hline dist $_{k e}$ & distance from distribution center $\mathrm{k}$ to store $\mathrm{e}$ \\
\hline
\end{tabular}

varying logistics costs for transferring the products between the entities of the supply chain and also production costs, as the objective function that needs to be minimised. Table 1 describes the nomenclature used in the model.

We develop a linear programming model as follows:

- The cost $Z$ is defined as:

$$
\begin{aligned}
& \operatorname{Minz}=\sum_{i} \sum_{j} x_{i j} c_{i j}+ \\
& \sum_{j}\left(c t f_{j}+c t v_{j}\left(\sum_{k} \operatorname{dist}_{j k}\left(\sum_{i} \operatorname{trans}_{i j k}\right)\right)\right)+ \\
& \sum_{k}\left(c t f_{k}+c t v_{k}\left(\sum_{e} \operatorname{dist}_{k e}\left(\sum_{i} \operatorname{trans}_{i k e}\right)\right)\right)
\end{aligned}
$$

In this function the first sum accounts for the cost of producing the designed cloths by the producers and the 2nd and the 3rd sums are accounting for the cost of transportation of the goods.

- The quantity of the produced goods must be less than their capacity:

$$
\sum_{i} x_{i j} \leq \operatorname{cap}_{j} \quad \forall j
$$

- The amount of transferred goods from the producer to the distribution center must be less than the available quantity of that product in the producer's warehouse:

$$
x_{i j}-\sum_{k} \operatorname{trans}_{i j k} \geq 0 \quad \forall i \forall j
$$

- The amount of transferred goods to the distribution centers must be less than their capacity:

$$
\sum_{i} \sum_{j} \operatorname{trans}_{i j k} \leq \operatorname{cap}_{k} \quad \forall k
$$

- The amount of goods transferred to the stores must be less than the amount of goods transferred from the 
manufacturer to the distribution center.

$$
\sum_{j} \operatorname{trans}_{i j k}-\sum_{e} \operatorname{trans}_{i k e} \geq 0 \quad \forall i \forall k
$$

- The amount of products transferred to the stores must be less than their capacity:

$$
\sum_{i} \sum_{k} \operatorname{trans}_{i k e} \leq \operatorname{cap}_{e} \quad \forall e
$$

- The amount of products transferred to the stores must must satisfy the demand:

$$
\sum_{k} \operatorname{trans}_{i k e} \geq \operatorname{dem}_{i e} \quad \forall i \forall e
$$

- Necessary boundaries:

$$
\operatorname{trans}_{i k e}, \operatorname{trans}_{i j k}, x_{i j} \geq 0
$$

\section{Model validation}

To validate the suggested model, historical data associated with costs and demands of the Queen Manto brand are being used. The direct supply chain of our problem consists of ten different designed products, three manufacturer, four distribution centers and three stores.

We used GAMS to solve the problem. The solver successfully converged under two seconds and found the optimal value of the objective function to be $Z=935209$. The decision variables values for the solution are given in figures $2-4$.

\section{Discussion}

Supply chain managers in the fashion industry have to make reasonable and fast decisions due to high demand and its complex dynamics. Our novel model can help the managers to make better decisions about their production, replenishment, and logistics policies in a reasonable time. As opposed to previous works such as [4] that was limited to only manufacturers and stores, our suggested model considers the decisions of designers, manufacturers, distribution centers and stores in a more comprehensive way.

We believe managers in the fashion industry equipped with our model and with an analytical point of view, can have better control over their supply chain components,

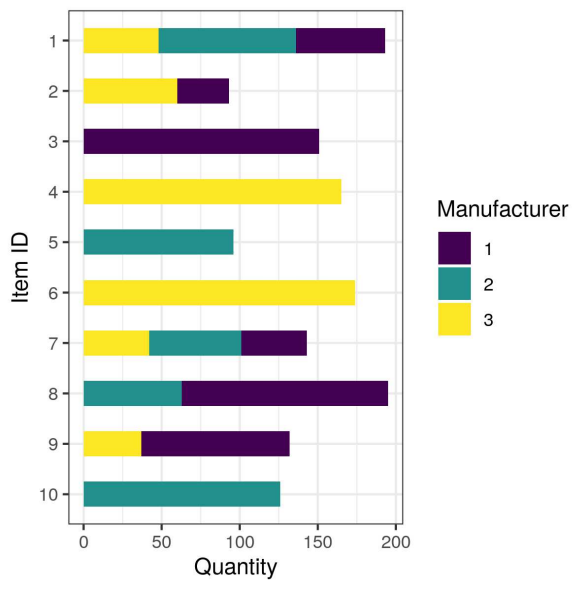

Fig. 2. The optimal quantity of item $i$ to be produced by manufacturer $j$.

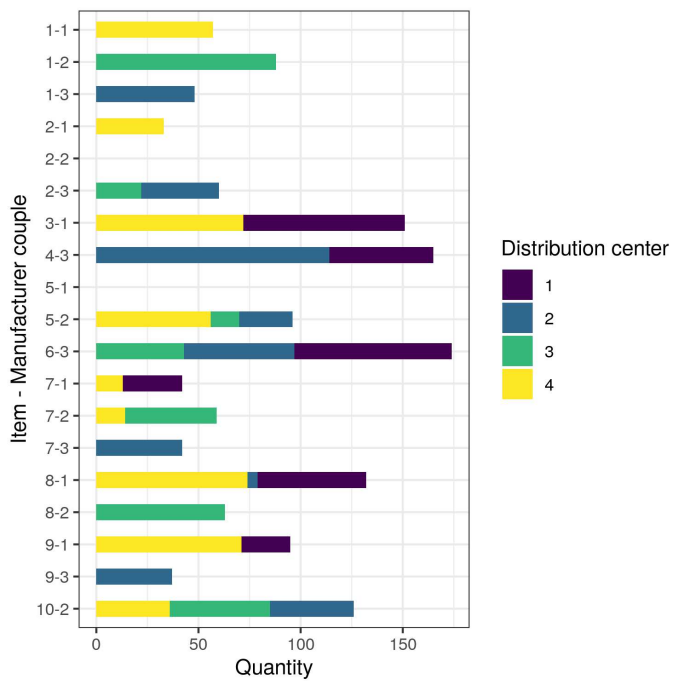

Fig. 3. The optimal quantity of item $i$ produced by manufacturer $j(i-j$ is coded on the y-axis) to be sent to distributor $k$.

such as logistics between different centers, and make decisions that result in more efficient use of resources. For instance, if the managers come to realize that the amount of goods transferred between two centers are frequently high in different periods, they might want to use more suitable and efficient transportation vehicles for those routes to further minimize their costs.

In summary, our model consisting of multiple levels from the designer to the customers can give valuable insights about the different parts of the supply chain and enable the decision makers to optimize their operations encompassing production quantities, logistics, inventory levels, and sales. However, our model has some limitations which we believe can be addressed by adding some extensions to it and making it more comprehensive and more flexible. Accordingly, future works could possibly include these extensions: considering dynamic pricing, 


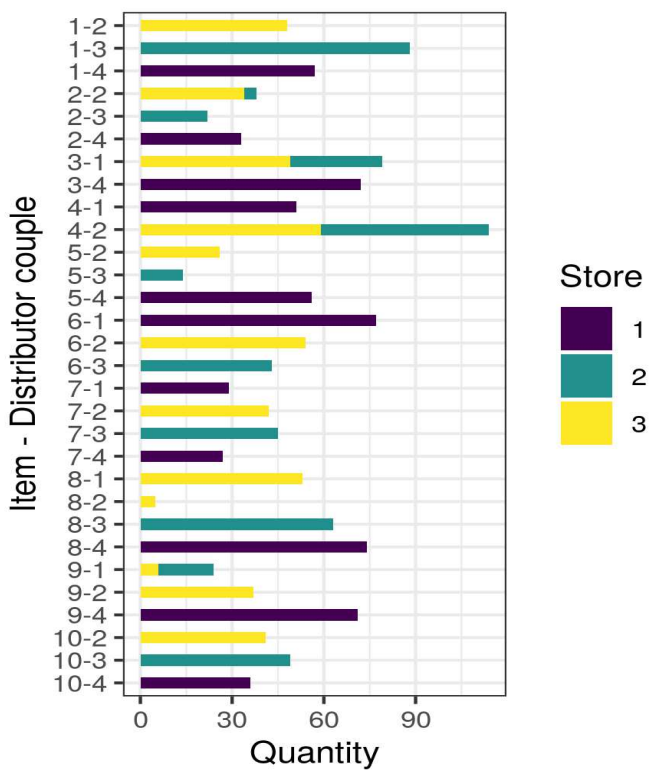

Fig. 4. The optimal quantity of item $i$ that distributor $k(i-k$ is coded on the y-axis) has to send to store $e$.

including promotions effects, and stochastic demand.

\section{Conclusion}

We developed a model to minimize production and logistics costs for fast response to customer demands and efficient replenishment in fashion supply chain with four major levels including designer,manufacturers, distribution centers, and stores. We validated our model using data from a fashion brand and solved for the optimal solution using GAMS. We reported the results of our model including the optimal quantity for production of the designers' designed items by each manufacturer, the optimal quantity of goods that need to be transferred from each manufacturer to each distribution center, and the optimal quantity of goods that need to be sent from the distribution centers to the stores in order to satisfy demand and minimise the costs.

\section{References}

[1] Reza Alikhani, S Ali Torabi, and Nezih Altay. Strategic supplier selection under sustainability and risk criteria. International Journal of Production Economics, 208:69-82, 2019.

[2] G. Blanchard and R. Loubere. Fashion industry, 2021. Available from: https://www.britannica.com/art/fashionindustry.

[3] Kasarin Charnsirisakskul, Paul M Griffin, and Pınar Keskinocak. Pricing and scheduling decisions with leadtime flexibility. European Journal of Operational Research, 171(1):153-169, 2006.

[4] Naila Fares, Maria Lebbar, and Najiba Sbihi. Quick response in fast fashion retail: An optimization supply chain responsiveness model. In 2018 4th International Conference on Optimization and Applications (ICOA), pages 1-5. IEEE, 2018.

[5] Marshall Fisher, Kumar Rajaram, and Ananth Raman. Optimizing inventory replenishment of retail fashion products. Manufacturing $\&$ service operations management, 3(3):230-241, 2001.

[6] Jérémie Gallien, Adam J Mersereau, Andres Garro, Alberte Dapena Mora, and Martín Nóvoa Vidal. Initial shipment decisions for new products at zara. Operations Research, 63(2):269-286, 2015.

[7] Yeu-Shiang Huang, Chia-Shuo Hsu, and Jyh-Wen Ho. Dynamic pricing for fashion goods with partial backlogging. International Journal of Production Research, 52(14):42994314, 2014.

[8] Raffaele Iannone, Giada Martino, Salvatore Miranda, and Stefano Riemma. Modeling fashion retail supply chain through causal loop diagram. IFAC-PapersOnLine, 48(3):1290-1295, 2015.

[9] Bob Lowson, Russell King, and Alan Hunter. Quick Response: Managing the supply chain to meet consumer demand. Wiley, 1999.

[10] Marzieh Mehrjoo and Zbigniew J Pasek. Risk assessment for the supply chain of fast fashion apparel industry: a system dynamics framework. International Journal of Production Research, 54(1):28-48, 2016.

[11] N Salehi Sadghiani, SA Torabi, and N Sahebjamnia. Retail supply chain network design under operational and disruption risks. Transportation Research Part E: Logistics and Transportation Review, 75:95-114, 2015. 